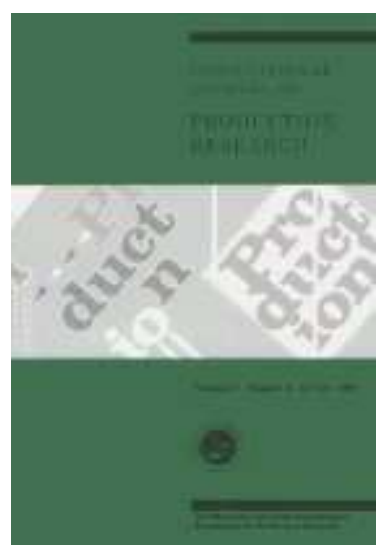

\title{
Storage policies and order picking strategies in U-shaped order-picking systems with a movable base
}

\begin{tabular}{|r|l|}
\hline Journal: & International Journal of Production Research \\
\hline Manuscript ID: & TPRS-2010-IJPR-1204 \\
\hline Manuscript Type: & Original Manuscript \\
\hline Author: & O6-Dec-2010 \\
\hline Complete List of Authors: & $\begin{array}{l}\text { Glock, Christoph; University of Wuerzburg, Chair of Business } \\
\text { Management and Industrial Management } \\
\text { Grosse, Eric; University of Wuerzburg, Chair of Business } \\
\text { Management and Industrial Management }\end{array}$ \\
\hline Keywords: & $\begin{array}{l}\text { ORDER PICKING METHODS, STORAGE SYSTEMS, WAREHOUSE } \\
\text { DESIGN, FACILITY LAYOUT }\end{array}$ \\
\hline Keywords (user): & \multicolumn{2}{|l}{} \\
\hline \multicolumn{2}{|l}{}
\end{tabular}

\section{SCHOLARONE"}

Manuscripts 


\title{
Storage policies and order picking strategies in U-shaped order-picking sys- tems with a movable base
}

\author{
Christoph H. Glock* \\ Eric H. Grosse \\ Chair of Business Management and Industrial Management \\ Faculty of Economics \\ University of Wuerzburg \\ Sanderring 2 \\ 97070 Wuerzburg, Germany
}

\begin{abstract}
Prior research on storage policies and order picking strategies in order picking systems has concentrated on studying warehouses with parallel shelves that are separated by horizontal and/or vertical aisles. This paper analyses a special case of an order picking system, where the warehouse is divided into zones with shelves being arranged in the shape of a $U$ in each of the zones. The paper assumes that the shelves of the order picking system are made up of two rows of stillages that can be flexibly exchanged and that the base of the order picking process can be moved within the aisle prior to the beginning of the order picking process. We describe the order picking system in a formal model and propose different storage location assignment policies whose efficiency is compared in a numerical study. The paper pays special attention to the practical applicability of the model and proposes heuristics that can be easily implemented in practice.
\end{abstract}

Keywords: order picking systems, warehouse layout, warehouse optimisation, storage location assignment

\footnotetext{
"Corresponding author. Tel.: ++49 931 3182408, Fax: ++49 931 312405, E-Mail: christoph.glock@uniwuerzburg.de.
} 


\section{Introduction}

Order picking, i.e. the process of retrieving items from their storage locations to fulfil a customer order, typically accounts for a high proportion of total warehouse operating costs (see e.g. Coyle et al. 1996, Frazelle 2002, Tompkins et al. 2003). The high cost impact of order picking is caused by the fact that orders are picked manually in many companies, which renders the travelling time of an order picker a critical factor in the overall efficiency of the warehouse (Bartholdi and Hackmann 2002). Changes in the competitive environment, such as high time pressure and variations in the demand pattern of the customers, induce companies to search for strategies that simultaneously reduce the costs and increase the speed of their order picking systems (Le-Duc and de Koster 2005). Researchers have proposed various strategies for designing the layout of a warehouse and for performing the order picking process and have shown that adopting a sophisticated order picking strategy may significantly reduce warehouse operating costs.

A closer look at the literature reveals that research in this area has concentrated on warehouses with parallel shelves that are separated by horizontal and/or vertical aisles. While this layout can be found in many warehouses, other setups for the order picking system may be beneficial in certain application areas. In this paper, we study a warehouse that is divided into zones with shelves being arranged in the shape of a $U$ in each of the zones. We assume that the shelves of the order picking system are made up of stillages that can be flexibly exchanged and that the base of the order picking process, i.e. the point where the order picker places the items retrieved from the warehouse, can be assigned to any place on the centre line of the aisle prior to the beginning of the order picking process. The objective of the paper is 1) to model this system, 2) to study how the position of the base influences system performance, and 3) to develop and compare different storage policies that help to reduce the average time the order picker needs to complete an order.

The remainder of the paper is organised as follows: The next two sections review related literature and provide a description of the problem under study. In section 4, we develop a mathematical model for a U-shaped order picking system with a movable base and conduct numerical experiments in section 5. The last section concludes the article and contains suggestions for future research.

\section{Literature review}

The order picking process has received a lot of attention in recent years. Papers that have been published in this area can roughly be differentiated into works that study the layout of the 
warehouse, works that analyse routing strategies in order picking systems and works that focus on the assignment of products to storage locations (for a review of literature on order picking systems, the reader is referred to de Koster et al. 2007).

The first stream of research analyses how the layout of the warehouse, i.e. the number and location of shelves and the number and dimension of aisles, influences the efficiency of the order picking system. Caron et al. (2000), for example, developed a model that helps to calculate the optimal number and length of aisles with the intention to minimise the expected tour distance for a given set of operating policies. A similar model was proposed by Roodbergen and Vis (2006), who further considered the location of the base as a decision variable in their analysis. Roodbergen et al. (2008), in turn, extended prior works by considering multiple blocks of parallel shelves and thus accounted for cross aisles as well.

The second stream of research studies routing strategies in order picking systems. The objective of works in this category is to determine the sequence in which items are retrieved from the warehouse with the intention to minimise the travel distance of the order picker. In this context, some authors tried to find the optimal picking route (see e.g. Ratliff and Rosenthal 1983, Goetschalckx and Ratliff 1988b), whereas others adopted heuristic routing policies (see e.g. Petersen and Schmenner 1999, Hall 1993). De Koster and van der Poort (1998), Hwang et al. (2004) and Petersen and Aase (2004), among others, compared different heuristic routing strategies for manual order picking systems and showed that heuristics may lead to good results, as compared to the optimal solution. Ratliff and Rosenthal (1983), in turn, formulated the order picking problem in a rectangular warehouse as a special case of the travelling salesman problem and provided a procedure for deriving a minimum length tour. The advantage of heuristic routing strategies is obviously that they are more straightforward and logical, less confusing and easier to follow for the picker (Petersen and Aase 2004, de Koster et al. 2007), wherefore some authors recommended their use in practice. In fact, some routing heuristics are able to generate near-optimal solutions and reduce the risk of picking failures as well (Petersen and Schmenner 1999).

A problem that is closely related to finding a tour of minimum length in an order picking system is commonly referred to as order batching. In this case, orders are either consolidated and picked by a single order picker or divided into sets which are subsequently picked by different pickers in separate zones of the warehouse. For an overview of order batching, the reader is referred to Gu et al. (2007), Bozer and Kile (2008) and Tsai et al. (2008), among others. 
The third research stream develops and compares strategies which determine how products should be assigned to the shelves of a warehouse with the intention of reducing the costs of retrieving the items from the shelves. In principle, three basic types of storage policies are differentiated in the literature (see e.g. de Koster et al. 2007, Gu et al. 2007, 2010): In case a random storage policy is used, products are assigned to storage locations without taking characteristics of the product into consideration. Thus, pallets arriving at the warehouse are assigned to a storage location selected randomly from all eligible empty locations with equal probability. This policy results in a high degree of storage space utilisation and is easy to implement, but usually leads to a high average travel distance for the order picker. In case of a dedicated storage policy, each product is stored in a fixed location of the warehouse. Usually, product characteristics, such as order picking frequency, weight or measurements (see Frazelle 2002, Brynzér and Johansson 1996) are used to determine the storage location of a product. For example, products with a high picking frequency could be stored close to the base to ensure that they can be picked without having to travel long distances in the warehouse (see e.g. Hausman et al. 1976, Dichtl and Beeskow 1980, Thonemann and Brandeau 1998, Brynzér and Johansson 1996). Although the degree of storage space utilisation is lower than in the case of a random storage policy, using a fixed location for products usually reduces the average travel time of the order picker. The third policy, which is commonly referred to as class-based storage, divides products into classes which are then stored in dedicated areas of the warehouse, whereby storage within an area is random. Obviously, classbased storage policies try to achieve a high degree of storage space utilisation by simultaneously reducing the average travel distance of the order picker.

The layout of automatic storage systems and the retrieval of items from storage locations is analysed in Park (2006), Yu and de Koster (2009) and Hu et al. (2009), among others, which shall not be discussed in greater detail here.

The discussion presented above illustrates that a variety of different problems has been analysed in the context of order picking systems. However, most works on order picking focus on warehouses with parallel shelves that are separated by horizontal and/or vertical aisles. In the following, we present a layout for an order picking system that uses U-shaped shelves made up of stillages. To the best of our knowledge, this layout has not been studied before.

\section{Problem description}

This paper studies order picking in a warehouse which is divided into zones with shelves being arranged in the shape of a $U$ in each of the zones, as is illustrated in Figure 1 (note that the 
zones are separated by dashed lines in the figure). We assume that the shelves of the order picking system are made up of stillages that are placed in two rows one above the other, and that one order picker is responsible for each of the zones. The stillages have an opening on one side facing the aisle which allows the order picker to remove items, and further they can be stacked. The order picking process is as follows: First, the order picker receives a customer order and places the base, which may be another stillage, for instance, on the centre line of the aisle (cf. Figure 1, where the base is represented by a black box, for some examples). The picker then starts retrieving items from the shelves until either the order is completed or his carrying capacity is reached, and then returns to the base to drop the items there. The order picker then continues to pick items from the shelves in this manner until the customer order is completed, and then removes the base from the zone so as to be ready to process another customer order. We assume that the base may only be moved at the beginning and the end of the order picking process due to weight reasons, and that a fork lifter or lifting cart is necessary to move it. In case one of the stillages is empty, the order picker has to replace it from the reserve area of the warehouse, which has to be done with the help of a fork lifter. It is obvious that it is easier to replace stillages in the upper row of the shelves, since replacing stillages from the lower row necessitates removing the upper stillage as well to get access to the stillage located below.

Figure 1

Order picking systems as the one described in this paper can be found in various application areas. For example, this layout is useful in cases where a workstation at an assembly line has to be supplied with different items that are to a great extent exclusively used at this work station, and where frequent orders to the warehouse are placed. In this case, it is beneficial to divide the warehouse into zones and use a separate zone for supplying each of the workplaces. Arranging the shelves in the shape of a $U$ and closing one end of the aisle, in this context, helps to reduce the travel distance of the order picker.

Using stillages instead of fixed racks increases the flexibility of the warehouse, since changes in the layout can be implemented quickly by re-positioning the stillages. Further, less investment is necessary since no additional racks have to be installed in the warehouse. However, this comes at the expense of higher operating costs due to the need to remove stillages 
from the upper row in case stillages in the lower row have to be replaced. One company we interviewed during our research on this model indicated that it used this type of setup temporarily to bridge the time until the completion of a new logistics centre. Due to the short period of use remaining for their current warehouse, the company chose not to invest in more sophisticated (and more expensive) warehouse equipment.

The objective of this paper is 1) to model this order picking system, 2) to study how the position of the base influences system performance, and 3 ) to develop and compare different storage policies that help to reduce the average time the order picker needs to complete an order. We study only a single zone of the order picking system since all zones operate independently of each other. The questions of where to locate which zone and how to direct the flows of materials in the warehouse are not addresses in this paper and are reserved for future research.

\section{Model development}

\subsection{Definitions}

Apart from the assumptions already stated, we assume the following hereafter:

- The stillages are numbered in an ascending order as shown in Figure 2, whereby 0 describes the base. Stillages in the upper row of the shelves are marked with 'up' in Figure 2 , while stillages in the lower row are marked with an 'lo'.

- We calculate Euclidean distances to estimate the travel distance between the base and a stillage or between any two stillages as we assume that this is the most intuitive way for the order picker to travel from one point of the order picking system to the next. However, since it has been shown by Goetschalckx and Ratliff (1988a) that the concept used for calculating travel distances does only slightly influence the performance of the system, rectangular distances, for example, could be used as well.

- Distances are measured from the centre of the base and from the centre of the front of the stillages (see Figure 2 for an example).

- The base can be moved on the centre line of the aisle before the start of the order picking process (see Goetschalckx and Ratliff 1988a for a similar assumption). One possible reason why the location of the base is restricted to the centre line is that it needs to be picked up with a fork lifter and that enough space has to be available on all sides to enable a smooth pick up-process. The distance between the centre of the base and the two parallel shelves thus equals $b / 2$. 
- We use a two-dimensional coordinate system to describe the location of the base and the stillages. The origin of the coordinate system is the outmost left position on the centre line of the aisle that the base can occupy, as is illustrated in Figure 2. If $x$ describes the position along the aisle and $y$ the distance from the centre line of the aisle, then the $y$ coordinate of the base is always 0 and the $y$-coordinate of the upper/lower shelve (as seen in Figure 2) is always $b / 2$ or $-b / 2$, respectively. Stillages that are placed on top of each other have the same coordinates.

- For the sake of simplicity, we assume that only complete picks are performed at a stillage. Consequently, the order picker will only move to a stillage if his carrying capacity is sufficient to pick all required items from the stillage. If the carrying capacity is not sufficient, the order picker will return to the base first before continuing the tour. Another consequence of this assumption is that the demand at none of the stillages exceeds the carrying capacity of the order picker, i.e. that $q_{i} \leq Q \forall i$.

- For the sake of brevity, we will use the male gender to refer to actors that could be male or female.

Figure 2

The following terminology will be used throughout the paper:

$a_{i, j, k} \quad$ decision variable which is 1 if the order picker travels from stillage $i$ to stillage $j$ in tour $k$ and which is 0 otherwise

$b \quad$ width of the aisle with $b=m w+(m+1) z$

$c_{i, k} \quad$ variable which is 1 if stillage $i$ is visited in tour $k$ and which is 0 otherwise

$D \quad$ travel distance of the order picker for completing a given picklist

$d_{i, j} \quad$ distance between stillages $i$ and $j$

$H \quad$ capacity of a stillage

$I_{i} \quad$ indices for the correlation-based assignment heuristic

$\bar{I}_{2} \quad$ cut off-level for the correlation-based assignment heuristic

$K \quad$ number of tours the order picker needs to complete a pick list

$l \quad$ length of the aisle $l=n w+(n-1) z$

$\lambda_{i} \quad$ average number of replacements of stillage $i$ per pick list

$m \quad$ number of stillages in the vertical shelve of a zone 
$\mu_{i} \quad$ average number of picks of item $i$ per pick list with $\left.\left.\mu_{i} \in\right] 0,1\right]$

$N \quad$ total number of boxes in a zone, with $N=2(2 n+m)$

$n \quad$ number of stillages in one of the two horizontal shelves of a zone

$P_{i} \quad$ average penalty costs for exchanging stillage $i$

$p \quad$ penalty cost value for exchanging stillages in the lower rows of the shelves

$Q \quad$ carrying capacity of the order picker

$Q^{u t i l} \quad$ carrying capacity used by the order picker

$q_{i} \quad$ cumulative weight of all items that need to be picked at stillage $i$. Obviously, $q_{i}=0$ $\forall i \notin \Omega$

$\sigma_{i, j} \quad$ measure for the correlation in demand between items $i$ and $j$ with $\sigma_{i, j} \in[0,1]$

$v \quad$ penalty cost value for moving the base along the centre line of the aisle

$w \quad$ width of a stillage

$x_{i} \quad$ coordinate that measures the position of the base or stillage $i$ along the centre line of the aisle

$x_{0, \max } \operatorname{maximum} x$-coordinate the base may adopt

$y_{i} \quad$ coordinate that measures the distance of the base or stillage $i$ from the centre line of the aisle

$z \quad$ gap between two stillages

$\Psi \quad$ the set of stillages with $\Psi=\{1, \ldots, N\}$

$\Omega \quad$ the set of stillages containing items that need to be picked with $\Omega \subseteq \Psi$

$M_{1} \quad$ the set of stillages in the upper rows of the parallel shelves with $M_{1}:=\{x \mid x=2 i-1, i=1, \ldots, 2 n\}$

$M_{2} \quad$ the set of stillages in the lower rows of the parallel shelves with $M_{2}:=\{x \mid x=2 i, i=1, \ldots, 2 n\}$

$M_{3} \quad$ the set of stillages in the upper rows of the rectangular shelve with $M_{3}:=\{x \mid x=4 n+2 i-1, i=1, \ldots, m\}$

$M_{4} \quad$ the set of stillages in the upper rows of the rectangular shelve with $M_{4}:=\{x \mid x=4 n+2 i, i=1, \ldots, m\}$

$\max [i]$ gives the order number of the stillage with the highest $x$-coordinate that contains items that need to be picked, i.e. $\max [i]:=\{i \in \mathbb{Z} \mid i=\operatorname{argmax}\{i \in \Omega\}\}$ 
To facilitate the development of the model, the problem under study will be divided into five sub-problems, namely a) the calculation of Euclidean distances, b) the routing problem, c) the determination of a location for the base, d) the problem of exchanging stillages and e) the storage location assignment problem. The sub-problems will be discussed separately in the following, and a summary will be presented in section 4.7 .

\subsection{Calculation of Euclidean distances}

As discussed above, the base is assumed to be located on the centre line of the aisle, and the order picker is assumed to travel in a straight line from the base to the stillages and between the stillages. If a stillage is $w$ meters wide with a gap of $z$ meters between any two stillages (which might be necessary to facilitate picking it up with a fork lifter), then the length of an aisle with $n$ stillages equals $l=n w+(n-1) z$ meters, while the width with $m$ stillages equals $b=$ $m w+(m+1) z$ meters (see Figure 2$)$. The coordinates of the base equal $\left(x_{0}, 0\right)$, while the coordinates of the stillages 1 to $4 n$ equal $(0, b / 2),(w+z, b / 2),(2(w+z), b / 2)$ etc. and $(0,-b / 2),(w+z,-$ $b / 2),(2(w+z),-b / 2)$ etc., respectively. The coordinates of the $j$ th stillage in the upper shelve are consequently given as $((j-1)(w+z), b / 2)$, while the coordinates of the $j$ th stillage in the lower shelve are given as $((j-1)(w+z),-b / 2)$. The coordinates of stillages $4 n+1$ to $N$ (i.e. the stillages in the right shelve, as seen in Figure 2) depend on the parameter $m$ : In case $m$ is even, the coordinates of the stillages are $(1-\mathrm{w} / 2,1 / 2(\mathrm{w}+\mathrm{z})),(1-\mathrm{w} / 2,3 / 2(\mathrm{w}+\mathrm{z}))$ etc. and $(1-\mathrm{w} / 2,-$ $1 / 2(\mathrm{w}+\mathrm{z})),(1-\mathrm{w} / 2,-3 / 2(\mathrm{w}+\mathrm{z}))$ etc., respectively, while in the odd case we have $(1-\mathrm{w} / 2,0)$ as well as $(1-\mathrm{w} / 2, \mathrm{w}+\mathrm{z}),(1-\mathrm{w} / 2,2(\mathrm{w}+\mathrm{z}))$ etc. and $(1-\mathrm{w} / 2,-(\mathrm{w}+\mathrm{z})),(1-\mathrm{w} / 2,-2(\mathrm{w}+\mathrm{z}))$ etc., respectively. The distance between any two points $i$ and $j$, i.e. between the base and a stillage or between any two stillages can consequently be calculated as follows (see Warnecke and Dangelmaier 1981).

$$
d_{i, j}=\sqrt{\left(x_{i}-x_{j}\right)^{2}+\left(y_{i}-y_{j}\right)^{2}}
$$

\subsection{The routing problem}

For a given storage policy and a given location of the base, the problem is to determine a sequence in which the order picker travels from the base to the stillages and back which minimises total travel distance. As the order picker has a limited carrying capacity and intends to visit each stillage only once per pick list, this problem is identical to the Capacitated Vehicle Routing Problem (CVRP) discussed in the literature, wherein a vehicle ships a product to a set of customers in $k$ tours (see e.g. Dantzig and Ramser 1959; Clarke and Wright 1964). If $d_{i, j}$ 
describes the distance between stillages $i$ and $j$ (or between the base and a stillage in case $i$ or $j$ equals 0), the objective function of the CVRP may be adopted as follows to suit the present problem:

$$
\min \sum_{k=1}^{K} \sum_{i \neq j} d_{i, j} a_{i, j, k}
$$

In addition, the following conditions have to be satisfied to guarantee that a feasible schedule is constructed.

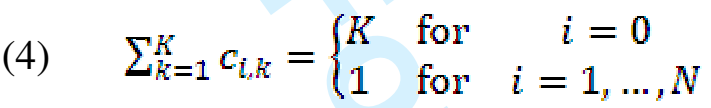

$$
\sum_{j=0}^{N} a_{i, j, k}=c_{i, k} \text { for } i=0, \ldots, N ; k=1, \ldots, K
$$$$
\sum_{i=0}^{N} a_{i, j, k}=c_{i, k} \text { for } j=0, \ldots, N ; k=1, \ldots, K
$$$$
\sum_{i, j \in H} a_{i, j, k} \leq|H|-1 \quad H \subseteq V ;|H| \geq 1, k=1, \ldots, K
$$

$$
a_{i, j, k} \in\{0,1\} \text { for } i, j=1, \ldots, N ; k=1, \ldots, K .
$$

$c_{i, k} \in\{0,1\}$ for $i=1, \ldots, N ; k=1, \ldots, K$.

Condition (3) ensures that the order picker does not exceed his carrying capacity when picking items, while (4) assures that each stillage is only visited once per pick list, with the exception that the base is visited $K$ times. (5) and (6) assign the stillages to the tours in which they are visited, (7) excludes short cycles from the analysis, and (8) and (9) ensure that the decision variables can only adopt the values 0 or 1 .

As to solution methods for the CVRP, researchers have concentrated on developing both exact and approximate algorithms for solving the problem due to its mathematical complexity (the CVRP belongs to the class of NP-hard problems; see Laporte 1992, Laporte and Semet 2002 and Cordeau et al. 2004 for a review of solution methods). In general, an increase in the quality of the solution, which can often be achieved with the help of an exact method, has to be balanced against increases in the run time of the algorithm, which may be especially critical in cases where results are needed immediately, as in the present problem. In addition, order pickers may deviate from a predefined route in case it appears illogical or suboptimal to them (see e.g. de Koster et al. 1999; Dekker et al. 2004; Gademann and van de Velde 2005), which may lead to high increases in the average travel distance. Since our paper emphasises the practical applicability of the model developed herein, we use a simple Sweep Algorithm (see e.g. Wren 1971; Wren and Holliday 1972; Gillett and Miller 1974) to construct a schedule for the order picker. The algorithm combines the advantages of a short run time with re- 
Step 0: Set $j^{\text {init }}=j=k=1, Q^{u t i l}=0, D^{*}=\infty, a_{i, j, k}=0 \forall i, j, k$ and $c_{i, k}=0 \forall i, k$.

Step 1: If $q_{j}>0$, set $a_{0, j, k}=c_{j, k}=1, j^{\text {last }}=j$ and $Q^{u t i l}=Q^{u t i l}+q_{j}$.

Else set $j=j+1$ and go to Step 1 .

Step 2: If $j=j^{\text {init }}-1$, go to Step 4 .

If $j^{\text {init }}=1$ and $j=N$, go to Step 4 .

If $j^{i n i t}>1$ and $j=N$, set $j=0$.

Set $j=j+1$. If $q_{j}=0$, go to Step 2 .

Step3: If $Q^{u t i l}+q_{j} \leq Q$, set $a_{j}$ last $_{j, k}=c_{j}$ last $_{k}=1, j^{\text {last }}=j$ and $Q^{u t i l}=Q^{u t i l}+q_{j}$ and go to Step 2 .

Else set $a_{j \text { last }, Q, k}=1, k=k+1$ and $Q^{u t i l}=0$ and go to Step 1 .

Step 4: Set $a_{j}$ last, $0, k=1$.

Calculate $D$. If $D<D^{*}$, set $D^{*}=D, a_{i, j_{r} k}^{*}=a_{i, j, k} \forall i, j, k$ and $c_{i, k}^{*}=c_{i, k} \forall i, k$.

If $j^{i n i t}<N$, set $j^{i n i t}=j^{i n i t}+1, j=j^{i n i t}, k=1, Q^{u t i l}=0, a_{i, j, k}=0 \forall i, j, k$ and $c_{i, k}=0 \forall i, k$ and go to Step 1.

After the algorithm terminates, the solution is given as $a_{i, k, k}^{*}$ and $c_{i, k}^{*}$ and the travel distance is given as $D^{*}$.

\subsection{Determination of a location for the base}

At the beginning of the order picking process, the order picker places the base somewhere on the centre line of the aisle. While the lowest possible value for the $x$-coordinate of the base is 0 , the highest possible value is determined by the $x$-coordinate of the stillage with the highest order number containing items that need to be picked, as is illustrated in appendix. The $x$ coordinate of the base may thus be chosen from the range $\left[0, x_{0, \max }\right]$. Increasing the $x$ coordinate of the base from 0 obviously serves the purpose to reduce the travel distance of the order picker. However, as the base has to be moved along the centre line, the order picker has to travel the distance $x_{0}$ twice when positioning the base, although he might move at a faster 
rate in case a fork lifter is used. The additional distance that has to be travelled when positioning the base has to be considered when formulating the objective function of the optimisation problem. Assuming that the order picker may move $2 v$ times as fast when placing the base at the centre line as compared to his walking speed in the order picking process, placing the base at position $\left(x_{0}, 0\right)$ requires the following time units:

(10) $\frac{2 x_{0}}{2 v}=\frac{x_{0}}{v}$

The following condition has to be considered:

$$
0 \leq x_{0} \leq x_{0, \max }
$$

\subsection{The problem of exchanging stillages}

As has been outlined above, the order picker is not only responsible for picking items from the stillages, but also for replacing stillages from the reserve area of the warehouse in case a stillage is depleted. In the following, we assume that the position of a stillage in the order picking system is irrelevant for calculating the travel distance between the zone and the reserve area of the warehouse, wherefore we only have to consider differences in the pick upprocess of the stillages (see Bhaskaran and Malmborg (1990) for a similar assumption). Since stillages are placed in two rows on top of each other to form the shelves of the order picking system, it is necessary to remove the stillage in the upper row as well if a stillage in the lower row is empty and needs to be replaced. After the order picker has brought a full stillage from the reserve area and placed it in the appropriate position in the zone, the stillage that has been removed before has to be put on top of the refilled stillage again. It is obvious that especially in a narrow aisle, where the order picker has to be careful when moving stillages, differences in the pick up-process of a stillage may significantly influence the average time to complete an order. If stillage $i$ needs to be exchanged on average $\lambda_{i}$ times per order picking process, and if exchanging a stillage in the lower row takes an equivalent of $p$ meters longer than exchanging a stillage in the upper row (i.e. the order picker could travel $p$ meters in the time difference between both pick up-processes), the following penalty value has to be considered when formulating the objective function of the optimisation problem:

$$
P_{i}=\left\{\begin{array}{ccc}
p \lambda_{i} & \text { for } & i \in M_{2} \cup M_{4} \\
0 & \text { for } & \text { else }
\end{array}\right.
$$

\subsection{The storage location assignment problem}

Apart from determining a location for the base and calculating a route for the order picker, the items need to be assigned to storage locations. The assignment of items to storage locations is 
specified in so-called storage policies (see Petersen and Schmenner 1999). In contrast to the two problems mentioned first, the assignment of items is not changed with every pick list, but remains constant for a longer period of time. Consequently, when determining rules of how to assign items to the stillages, characteristics of the demand situation as a whole, and not only of a particular pick list, have to be taken into consideration.

As has been outlined in the literature review, a variety of different storage policies has been analysed in the literature, including random, dedicated and class-based storage. In the following, we assume that a dedicated storage policy is used for assigning items to the stillages. Since determining an optimal storage policy is prohibitive due to the complexity of the problem, we adopt an approach that is commonly used in the literature and use a set of heuristic rules to assign items to the stillages. The heuristics are compared in section 5 in a simulation study to assess their performance. Specifically, we use the following heuristics to assign items to the stillages:

I) Random assignment: Items are randomly assigned to storage spaces, but the assignment is not changed afterwards. We use this rule as a benchmark to assess the performance of the other heuristics.

II) Horizontal assignment: Items are assigned to storage spaces according to their average number of picks. Starting with the item with the highest pick frequency and continuing with the item that is ranked second etc., items are assigned to the stillages in one of the parallel shelves of the aisle (cf. part a) of Figure 3 for an example, where A, B and C illustrate zones of the order picking system that are filled with decreasing priority; for other zoning heuristics, the reader is referred to Petersen $(1999,2002)$ and Petersen et al. (2004), among others). The assignment starts in the utmost left stillage of the shelf and continues until the shelf has been completely filled. Thereby, items are always assigned to the upper stillage before an item is placed in the lower stillage as well. Subsequently, the second parallel shelf is filled, before items are assigned to the shelf on the right side of the aisle. We assume that this heuristic is especially beneficial in order picking systems with a wide aisle, since the order picker has to cross the aisle less frequently in such a setting.

III) Vertical assignment: As in the case of horizontal assignment, items are assigned to storage spaces according to their average number of picks. The difference between this heuristic and the one discussed under II) is that the items are not assigned to a single shelf until the shelf has been filled, but that items are allocated equally to both parallel shelves before moving down the aisle. Consequently, the order picking system is not zoned horizontally in this case, but vertically, as can be seen in part b) of Figure 3. We assume that this 
heuristic is especially beneficial in order picking systems with a narrow and long aisle, since the order picker has to travel less frequently down the aisle in such a setting.

IV)Upper/lower assignment: Again, items are assigned to storage spaces according to their average number of picks. In contrast to the heuristics discussed under II) and III), the stillages in the upper row of the shelves are filled first before items are assigned to stillages in the lower row (cf. part c) of Figure 3). To illustrate this heuristic, we combine it with the rule presented under II) and assume that the parallel shelves are filled before items are placed in the shelf on the right side of the aisle. We assume that this heuristic is especially beneficial in order picking systems where exchanging stillages in the lower row is costly, i.e. in systems with a high $p$-value.

V) Correlation-based assignment: The last heuristic considers both the average number of picks and the fact that the demands for two or more items may be correlated. As Dichtl and Beeskow (1980), Frazelle and Sharp (1989) and Liu (1999), among others, point out, correlations may occur in the demand of two or more items, which entails that the respective items are frequently demanded together. In such a case, it may be beneficial to place correlated items next to each other to avoid that the order picker has to travel large distances to retrieve them. In the following, we use two indices for assigning items to storage locations, which takes account of the fact that both correlation in demand and pick frequency are important for determining the storage location of an item. The two indices are calculated as follows:

$$
\begin{aligned}
& I_{1}=\mu_{i}\left(1+\sum_{j \backslash i} \sigma_{i, j}\right) \\
& I_{2}=\mu_{j}\left(1+\sigma_{i, j}\right)
\end{aligned}
$$

The parameter $\sigma_{i, j}$ is calculated as proposed by Frazelle and Sharp (1989) by counting the number of pick lists in which items $i$ and $j$ are requested together, and by dividing the resulting number by the total number of pick lists.

The heuristic works as follows: First, $I_{1}$ is calculated for each item, and all items are sorted in decreasing order of $I_{1}$. The item with the highest $I_{1}$-value is assigned to the first stillage in the upper row of the upper parallel shelf (i.e. to stillage 1 as seen in Figure 2). Accordingly, $I_{2}$ is calculated for all remaining items, and the items are sorted in decreasing order of $I_{2}$. All items that exceed the cut off-level $\bar{I}_{2}$ are assigned in decreasing order of their $I_{2}$-value to the stillages below or next to the one that has been reserved with the first product. Subsequently, the item that ranks next on the $I_{1}$-index (and has not yet been assigned to a stillage) is selected etc. until all items have been assigned to the storage loca- 


\subsection{Summary}

The preceding sections outlined the problem studied in this paper. The objective function of the problem may now be formulated by summing up expressions (2), (10) and (12), whereby (12) has to be summed up over all stillages:

$$
D=\min \left(\sum_{k=1}^{K} \sum_{i \neq j} d_{i, j} a_{i, j, k}+\frac{x_{0}}{v}+\sum_{i=1}^{N} P_{i}\right)
$$

In optimising expression (15), conditions (3) to (9) and (11) have to be considered. We solve the problem by first assigning items to storage locations and by then constructing tour schedules for the order picker for the case $x_{0}=0$. Subsequently, (15) is minimised with respect to $x_{0}$ to find an optimal location for the base, and the Sweep Algorithm is run again to update the tour length. This step is repeated until no further changes in the location of the base occur.

\section{Numerical examples}

To illustrate the behaviour of our model and to assess the efficiency of the storage policies proposed in section 4 , we conducted an extensive numerical study wherein we analysed the performance of the system for five different layouts of the warehouse, as illustrated in Table 1. The capacity of the order picking systems studied remained constant at 44 items for all problem instances, but the shape of the aisle was assumed to be different for each problem. While problem \#1 represents the case of a long and narrow aisle, problem \#5 stands for a short and wide aisle, and problems \#2 to \#4 for intermediate cases. As indicated above, it may be expected that the layout of the order picking system influences the efficiency of the storage policies proposed in section 4 .

Table 1 
To gain insight into the behaviour of our model, we tested 1,500 randomly generated data sets for each of the setups presented in Table 1, five alternative storage policies, four cut off-levels and three picking environments, leading to a total of 180,000 picklists that had to be computed. The solution algorithm was programmed in Mathematica 7.0 by Wolframs Research Inc., and the necessary data was generated by 1) computing a random binary variable which indicated whether the item was included on a pick list or not, 2) randomly selecting a demand from the range [1,5] and 3) calculating a random variable which led to correlations in the demand of the items. The other parameter values used in the numerical study were assumed as follows: $w=1.3, z=0.5, p=5, Q=15, v=3$ and $H=50$. As to the penalty cost value for moving the base along the centre line of the aisle, we tested two alternative values, $p$ $=5$ and $p=15$, and considered four alternative values for the cut off-level, $\bar{I}_{2}=0.5, \bar{I}_{2}=0.6$, $\bar{I}_{2}=0.7$ and $\bar{I}_{2}=0.8$.

Table 2 illustrates the average time being necessary to complete a picklist for the case where the base is fixed to $x_{0}=0$ and where penalty costs for exchanging lower stillages are low with $p=3$. As can be seen, the random storage policy $(\mathrm{R})$ led to the highest average travel time for all layouts studied in this case. Further, it can be seen that using a horizontal storage policy $(\mathrm{H})$ outperformed the vertical storage policy $(\mathrm{V})$ for the first three layouts, but that the vertical storage policy led to better results as the aisle became very narrow. The correlation-based assignment policy (C) outperformed all other heuristics, although its relative advantage as compared to the vertical assignment policy was reduced as the width of the aisle decreased. This is due to the fact that correlation-based assignment employed a horizontal assignment strategy in our simulation study, which increased travel time for the order picker as the aisle became narrower. However, we note that correlation-based assignment can be combined with a vertical assignment strategy as well, which should lead to better results for narrow aisles. Finally, table 2 illustrates that upper/lower assignment (U/L) only outperformed the random assignment policy in this case, which obviously was caused by the relatively low penalty cost value $v$.

Table 2

In a second step, we permitted the base to be moved along the centre line of the aisle before the start of the order picking process. The results presented in table 3 illustrate that the 
relative advantage of the storage policies was not changed by permitting $x_{0}$ to be varied, but that the average travel distance could be reduced for all policies. It can further be seen that for wide aisles, the base is moved relatively deep into the aisle to reduce the distance between the base and the shelf which closes the aisle on one side. As the length of the aisle increases, moving the base deep into the aisle leads to a high increase in penalty costs, wherefore a location closer to the open end of the aisle is preferred.

\section{Table 3}

In a last step, we increase the penalty cost value for exchanging lower stillages from 5 to 15 . Table 4 illustrates that an increase in $p$ leads to a higher average travel time for the order picker for all layouts and storage policies studied. However, it can be seen that the relative advantage of the upper/lower assignment policy increased as lower stillages had to be exchanged less frequently in this case. However, the correlation-based assignment policy still led to the best results.

Table 4

As to possible savings in total travel time, our examples illustrate that allowing the base to be moved along the centre line of the aisle and using one of the storage policies proposed in this paper reduces the average time the order picker needs to complete an order between $21.36 \%$ and $35.76 \%$ as compared to a random storage assignment and a fixed base. Even if the base is assigned to a fixed location, using one of the storage policies proposed in this paper reduced average picking time between $17.37 \%$ and $30.42 \%$. It is obvious that these savings may strongly increase the performance of the warehouse and improve the overall cost position of the company. As to the cut off-level for the correlation-based storage policy, $\bar{I}_{2}=$ 0.7 led to the best results for all data sets we studied. We note, however, that the impact of $\bar{I}_{2}$ depends on the characteristics of the data used, wherefore we recommend testing several alternative values in practice. 


\section{Conclusion}

This paper developed a model for a U-shaped order picking system and analysed how the location of the base and the assignment of products to the shelves of the warehouse impact the performance of the system. It could be shown that allowing the base to be moved along the aisle and using one of the storage policies proposed in this paper may reduce the average time which is needed to complete an order comprehensively. Numerical studies indicated that assigning products to the shelves in a horizontal manner is especially beneficial in wide aisles, while using a vertical storage assignment policy leads to better solutions in case aisles are narrow. Storage policies that consider demand correlations of products constantly led to better results in the problem instances studied in this paper.

The findings of this paper have several implications for practitioners: First, the paper proposed an alternative structure for order picking systems that had not been analysed in the literature before. This layout may be employed in several scenarios, for example in cases where different zones of a warehouse exclusively supply a workplace at an assembly line. Second, the paper proposed alternative strategies for assigning products to shelves in a Ushaped order picking system. Due to their relatively simple structure, the heuristic methods proposed in this paper can be directly applied in practice and thus help to increase the efficiency of order picking systems. Finally, we identified factors that influence the efficiency of the storage policy and were thus able to give decision support to practitioners.

One limitation of our paper clearly is that it only studied a single zone of the warehouse. Although the assumption that certain products are stored exclusively in a particular zone may hold in many practical scenarios, the location of the zone in the warehouse has a critical impact on the efficiency of the order picking system as it influences transportation times between the zone and the workplace as well as between the zone and the reserve area of the warehouse. Consequently, future research could concentrate on studying U-shaped order picking zones in the context of a warehouse with multiple zones. Further, it would be interesting to compare a warehouse consisting of U-shaped zones with the classical approach that employs horizontal and vertical aisles to study under which condition U-shaped zones provide efficiency gains. It is obvious that in case of very long aisles, inserting passages between the shelves may lead to performance improvements.

\section{Appendix}

Figure A-1 illustrates a section of the order picking system with two potential locations for the base and one stillage, denoted as $i$, out of which items need to be withdrawn. If we assume that stillage $i$ is the stillage with the highest order number which contains items that need to be 


\section{References}

Bartholdi, J.J. and Hackman S.T., 2002. Warehouse \& Distribution Science (accessible online under www.warehouse-science.com).

Bhaskaran, K. and Malmborg, C.J., 1990. Economic tradeoffs in sizing warehouse reserve storage area. Applied Mathematical Modelling, 14 (6), 381-385.

Bozer, Y.A. and Kile, J.W., 2008. Order batching in walk-and-pick order picking systems. International Journal of Production Research, 46 (7), 1887-1909.

Brynzér, H. and Johansson, M.I., 1996. Storage location assignment: Using the product structure to reduce order picking times. International Journal of Production Economics, 46-47 (1), 595-603. 
Caron, F., Marchet, G., and Perego, A., 2000. Optimal layout in low-level picker-to-part systems. International Journal of Production Research, 38 (1), 101-118.

Clarke, G. and Wright, J.W., 1964. Scheduling of vehicles from a central depot to a number of delivery points. Operations Research, 12 (4), 568-581.

Cordeau, J.F., Gendreau, M., Hertz, A., Laporte, G., and Sormany, J.-S., 2004. New Heuristics for the Vehicle Routing Problem. In: Langevin, A. and Riopel, D., eds. Logistic Systems - Design and Optimization. New York: Springer, 279-297.

Coyle, J.J., Bardi, E.J., and Langley, C.J., 1996. The Management of Business Logistics. St. Paul, MN: West.

Dantzig, G.B. and Ramser, J.H., 1959. The Truck Dispatching Problem. Management Science, 6 (1), 80-91.

De Koster, R., Le-Duc, T., and Roodbergen, K.J., 2007. Design and control of warehouse order picking: a literature review. European Journal of Operational Research, 182 (2), 481-501.

De Koster, R., Roodbergen, K.J., and van Voorden, R., 1999. Reduction of walking time in the distribution center of De Bijenkorf. In: Speranza, M.G. and Stähly, P., ed. New trends in distribution and logistics, Berlin: Springer, 215-234.

De Koster, R. and van der Poort, E.S., 1998. Routing orderpickers in a warehouse: a comparison between optimal and heuristic solutions. IIE Transactions, 30 (5), 469-480.

Dekker, R., de Koster, M.B.M., Roodbergen, K.J., and van Kalleveen, H., 2004. Improving Order-Picking Response Time at Ankor's Warehouse. Interfaces, 34 (4), 303-313.

Dichtl, E. and Beeskow, W., 1980. Die optimale Anordnung von Gütern in Vorratslägern mit Hilfe der Mehrdimensionalen Skalierung. Zeitschrift für Operations Research, 24 (2), B51-B64.

Frazelle, E.A., 2002. World-class warehousing and material handling. New York: McGrawHill.

Frazelle, E.A. and Sharp, G.P., 1989. Correlated assignment strategy can improve any orderpicking operation. Industrial Engineering, 21 (4), 33-37.

Gademann, N. and van de Velde, S., 2005. Order batching to minimize total travel time in a parallel-aisle warehouse. IIE Transactions, 37 (1), 63-75.

Gillet, B.E. and Miller, L.R., 1974. A heuristic algorithm for the vehicle-dispatch problem. Operations Research, 22 (2), 568-581.

Goetschalckx, M. and Ratliff, H.D., 1988a. An efficient algorithm to cluster order picking items in a wide aisle. Engineering Costs and Production Economics, 13 (3), 263-271. 
Goetschalckx, M. and Ratliff, H.D., 1988b. Order picking in an aisle. IIE Transactions, 20 (1), 53-62.

Gu, J., Goetschalckx, M., and Mc Ginnis, L.F., 2007. Research on warehouse operation: a comprehensive review. European Journal of Operational Research, 177 (1), 1-21.

Gu, J., Goetschalckx, M., and Mc Ginnis, L.F., 2010. Research on warehouse design and performance evaluation: a comprehensive review. European Journal of Operational Research, 203 (3), 539-549.

Hall, R.W., 1993. Distance approximations for routing manual pickers in a warehouse. IIE Transactions, 25 (4), 76-87.

Hausman, W.H., Schwarz, L.B., and Graves, S.C., 1976. Optimal storage assignment in automatic warehousing systems. Management Science, 22 (6), 629-638.

Hu, K., Chang, T., Fu, H., and Yeh, H., 2009. Improvement order picking in mobile storage systems with a middle cross aisle. International Journal of Production Research, 47 (4), 1089-1104.

Hwang, H., Oh, Y.H. and Lee, Y.K., 2004. An evaluation of routing policies for order-picking operations in low-level picker-to-part system. International Journal of Production Research, 42 (18), 3873-3889.

Laporte, G., 1992. The Vehicle Routing Problem: An overview of exact and approximate algorithms. European Journal of Operational Research, 59 (3), 345-358.

Laporte, G. and Semet, F., 2002. Classical Heuristics for the Capacitated VRP. In: Toth, P. and Vigo, D., eds. The Vehicle Routing Problem. Philadelphia: Siam, 109-126. 2002

Le-Duc, T. and de Koster, R., 2005. Travel distance estimation and storage zone optimization in a 2-block class-based storage strategy warehouse. International Journal of Production Research, 43 (17), 3561-3581.

Liu, C.-M., 1999. Clustering techniques for stock location and order-picking in a distribution center. Computers \& Operations Research, 26 (10-11), 989-1002.

Park, B.C., 2006. Performance of automated storage/retrieval systems with non-square-intime racks and two-class storage. International Journal of Production Research, 44 (6), 1107-1123.

Petersen, C.G., 1999. The impact of routing and storage policies on warehouse efficiency. International Journal of Operations \& Production Management, 19 (10), 1053-1064.

Petersen, C.G., 2002. Considerations in order picking zone configuration. International Journal of Operations \& Production Management, 22 (7), 793-805. 
Petersen, C.G. and Aase, G., 2004. A comparison of picking, storage, and routing policies in manual order picking. International Journal of Production Economics, 92 (1), 11-19.

Petersen, C.G. and Schmenner, R.W., 1999. An Evaluation of Routing and Volume-based Storage Policies in an Order Picking Operation. Decision Sciences, 30 (2), 481-501.

Ratliff, H.D. and Rosenthal, A.S., 1983. Orderpicking in a rectangular warehouse: a solvable case of the traveling salesman problem. Operations Research, 31 (3), 507-521.

Roodbergen, K.J., Sharp, G.P., and Vis, I.F.A., 2008. Designing the layout structure of manual order picking areas in warehouses. IIE Transactions, 40 (11), 1032-1045.

Roodbergen, K.J. and Vis, I.F.A., 2006. A model for warehouse layout. IIE Transactions, 38 (10), 799-811.

Thonemann, U.W. and Brandeau, M.L., 1998. Note: optimal storage assignment policies for automated storage and retrieval systems with stochastic demands. Management Science, 44 (1), 142-148.

Tompkins, J.A., White, J.A., Bozer, Y.A., and Tanchoco, J.M.A., 2003. Facilities Planning, 3rd ed. New Jersey: Wiley.

Tsai, C.-Y., Liou, J.J.H. and Huang, T.-M., 2008. Using a multiple-GA method to solve the batch picking problem: considering travel distance and order due time. International Journal of Production Research, 46 (22), 6533-6555.

Warnecke, H.J. and Dangelmeier, W., 1981. Layoutplanung - Stand der Technik. OR Spektrum, 3 (1), 1-20.

Wren, A., 1971. Computers in Transport Planning and Operation. London: Ian Allan.

Wren, A. and Holliday, A., 1972. Computer Scheduling of Vehicles from One or More Depots to a Number of Delivery Points. Operational Research Quarterly, 23 (3), 333344.

Yu, Y. and de Koster, M.B.M., 2009. Designing an optimal turnover-based storage rack for a 3D compact automated storage and retrieval system. International Journal of Production Research, 47 (6), 1551-1571.

\section{Captions for Figures and Tables}

Figure 1: Schematic view of an order picking system with U-shaped shelves

Figure 2: Example of a zone of the warehouse with 38 stillages

Figure 3: Illustration of the storage policies

Figure A-1: A sample aisle with two alternative locations for the base 
Table 1: Alternative layouts for the order picking system analysed in the numerical study Table 2: Average time to complete a picklist for a fixed base and $p=5$

Table 3: Average time to complete a picklist for a movable base and $p=5$

Table 4: Average time to complete a picklist for a movable base and $p=15$ 


\begin{tabular}{|c|c|c|c|c|c|}
\hline$\#$ & $\mathbf{1}$ & $\mathbf{2}$ & $\mathbf{3}$ & $\mathbf{4}$ & $\mathbf{5}$ \\
\hline $\boldsymbol{n}$ & 10 & 9 & 8 & 7 & 6 \\
\hline $\boldsymbol{m}$ & 2 & 4 & 6 & 8 & 10 \\
\hline $\boldsymbol{l}$ & 17.5 & 15.7 & 13.9 & 12.1 & 10.3 \\
\hline $\boldsymbol{b}$ & 4.1 & 7.7 & 11.3 & 14.9 & 18.5 \\
\hline
\end{tabular}

\section{Table 1}




\begin{tabular}{|l|c|c|c|c|c|}
\hline \multicolumn{1}{|c|}{ Type } & $\boldsymbol{D}_{\boldsymbol{a v}} \mathbf{R}$ & $\boldsymbol{D}_{\boldsymbol{a v}} \mathbf{H}$ & $\boldsymbol{D}_{\boldsymbol{a v}} \mathbf{V}$ & $\boldsymbol{D}_{\boldsymbol{a v}} \mathbf{U} / \mathbf{L}$ & $\boldsymbol{D}_{\boldsymbol{a v}} \mathbf{C}$ \\
\hline$n=6, m=10$ & 270.85 & 210.14 & 214.91 & 223.79 & 203.84 \\
\hline$n=7, m=8$ & 259.01 & 197.74 & 199.99 & 206.42 & 189.83 \\
\hline$n=8, m=6$ & 239.84 & 179.23 & 179.24 & 186.30 & 172.06 \\
\hline$n=9, m=4$ & 210.94 & 153.46 & 153.00 & 160.92 & 147.33 \\
\hline$n=10, m=2$ & 176.39 & 127.85 & 124.78 & 131.70 & 122.74 \\
\hline
\end{tabular}

\section{Table 2}




\begin{tabular}{|l|c|c|c|c|c|c|c|c|c|c|}
\hline \multirow{2}{*}{ Type } & \multicolumn{2}{|c|}{$\mathbf{R}$} & \multicolumn{2}{c|}{$\mathbf{H}$} & \multicolumn{2}{c|}{$\mathbf{V}$} & \multicolumn{2}{c|}{ U/L } & \multicolumn{2}{c|}{ C } \\
\cline { 2 - 12 } & $\boldsymbol{D}_{\boldsymbol{a v}}$ & $\boldsymbol{x}_{\mathbf{0}}$ & $\boldsymbol{D}_{\boldsymbol{a v}}$ & $\boldsymbol{x}_{\mathbf{0}}$ & $\boldsymbol{D}_{\boldsymbol{a v}}$ & $\boldsymbol{x}_{\mathbf{0}}$ & $\boldsymbol{D}_{\boldsymbol{a v}}$ & $\boldsymbol{x}_{\mathbf{0}}$ & $\boldsymbol{D}_{\boldsymbol{a v}}$ & $\boldsymbol{x}_{\mathbf{0}}$ \\
\hline$n=6, m=10$ & 253.02 & 6.97 & 202.15 & 5.90 & 206.72 & 5.94 & 212.99 & 6.55 & 198.10 & 6.79 \\
\hline$n=7, m=8$ & 238.55 & 7.38 & 187.99 & 6.07 & 190.98 & 6.18 & 194.60 & 6.81 & 180.47 & 7.21 \\
\hline$n=8, m=6$ & 217.13 & 7.28 & 169.06 & 5.94 & 169.74 & 6.08 & 174.23 & 6.72 & 163.98 & 7.26 \\
\hline$n=9, m=4$ & 163.75 & 8.49 & 142.25 & 5.80 & 143.14 & 5.81 & 148.57 & 6.54 & 139.40 & 7.18 \\
\hline$n=10, m=2$ & 156.59 & 6.86 & 115.63 & 5.75 & 113.58 & 5.49 & 117.25 & 6.23 & 109.85 & 7.03 \\
\hline
\end{tabular}

Table 3 


\begin{tabular}{|l|c|c|c|c|c|c|c|c|c|c|}
\hline \multirow{2}{*}{ Type } & \multicolumn{2}{|c|}{$\mathbf{R}$} & \multicolumn{2}{c|}{$\mathbf{H}$} & \multicolumn{2}{c|}{$\mathbf{V}$} & \multicolumn{2}{c|}{ U/L } & \multicolumn{2}{c|}{ C } \\
\cline { 2 - 11 } & $\boldsymbol{D}_{\boldsymbol{a v}}$ & $\boldsymbol{x}_{\mathbf{0}}$ & $\boldsymbol{D}_{\boldsymbol{a v}}$ & $\boldsymbol{x}_{\mathbf{0}}$ & $\boldsymbol{D}_{\boldsymbol{a v}}$ & $\boldsymbol{x}_{\mathbf{0}}$ & $\boldsymbol{D}_{\boldsymbol{a v}}$ & $\boldsymbol{x}_{\mathbf{0}}$ & $\boldsymbol{D}_{\boldsymbol{a v}}$ & $\boldsymbol{x}_{\mathbf{0}}$ \\
\hline$n=6, m=10$ & 273.42 & 6.97 & 207.97 & 5.90 & 212.63 & 5.94 & 213.31 & 6.55 & 201.73 & 6.79 \\
\hline$n=7, m=8$ & 246.90 & 7.38 & 193.80 & 6.07 & 196.82 & 6.18 & 195.2 & 6.81 & 186.05 & 7.21 \\
\hline$n=8, m=6$ & 223.88 & 7.28 & 174.09 & 5.94 & 175.56 & 6.08 & 175.13 & 6.72 & 170.61 & 7.26 \\
\hline$n=9, m=4$ & 189.24 & 8.49 & 148.21 & 5.80 & 148.96 & 5.81 & 150.00 & 6.54 & 143.76 & 7.18 \\
\hline$n=10, m=2$ & 148.29 & 6.86 & 121.49 & 5.75 & 119.41 & 5.49 & 119.11 & 6.23 & 119.06 & 7.03 \\
\hline
\end{tabular}

Table 4 


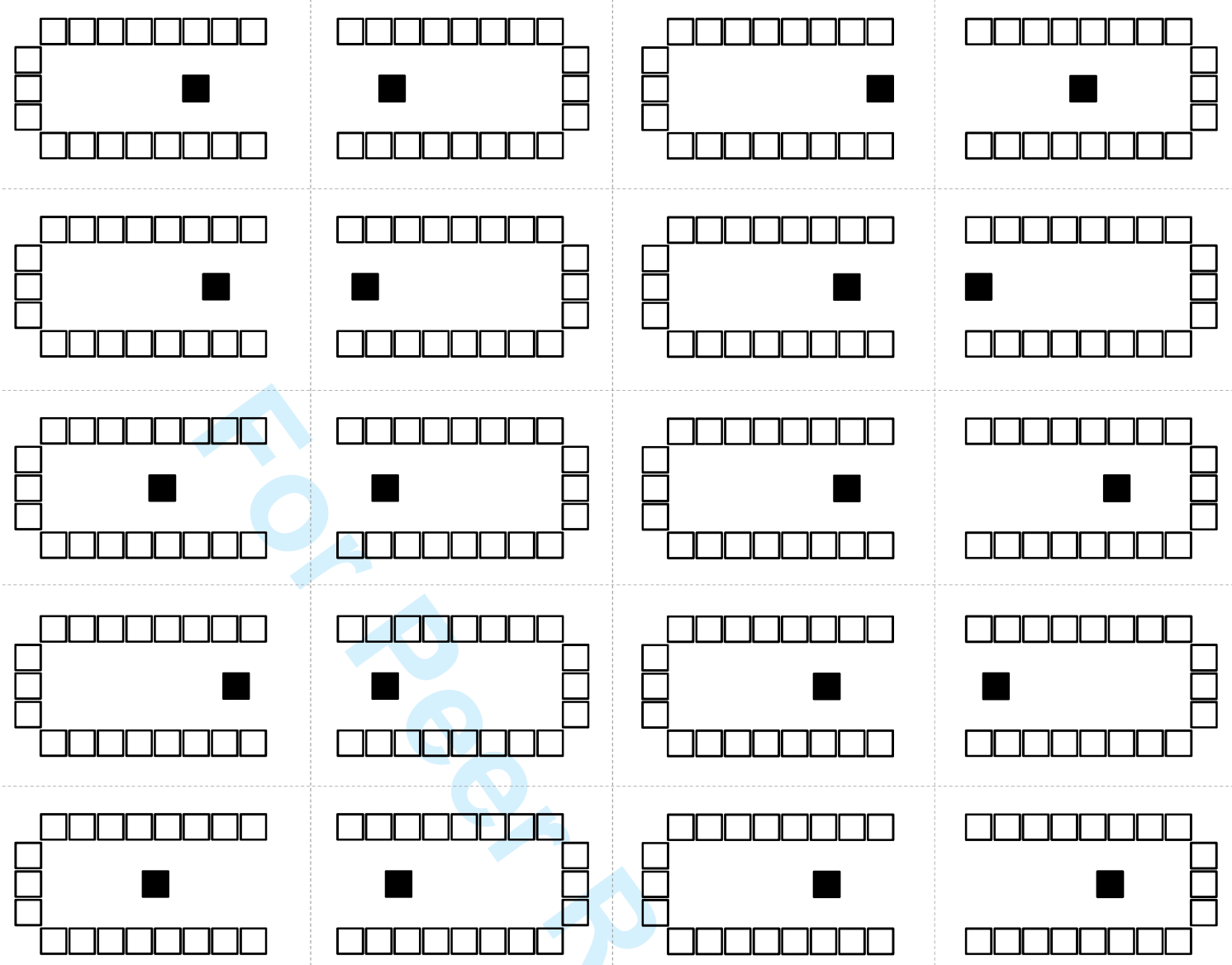

Figure 1: Schematic view of an order picking system with U-shaped shelves 


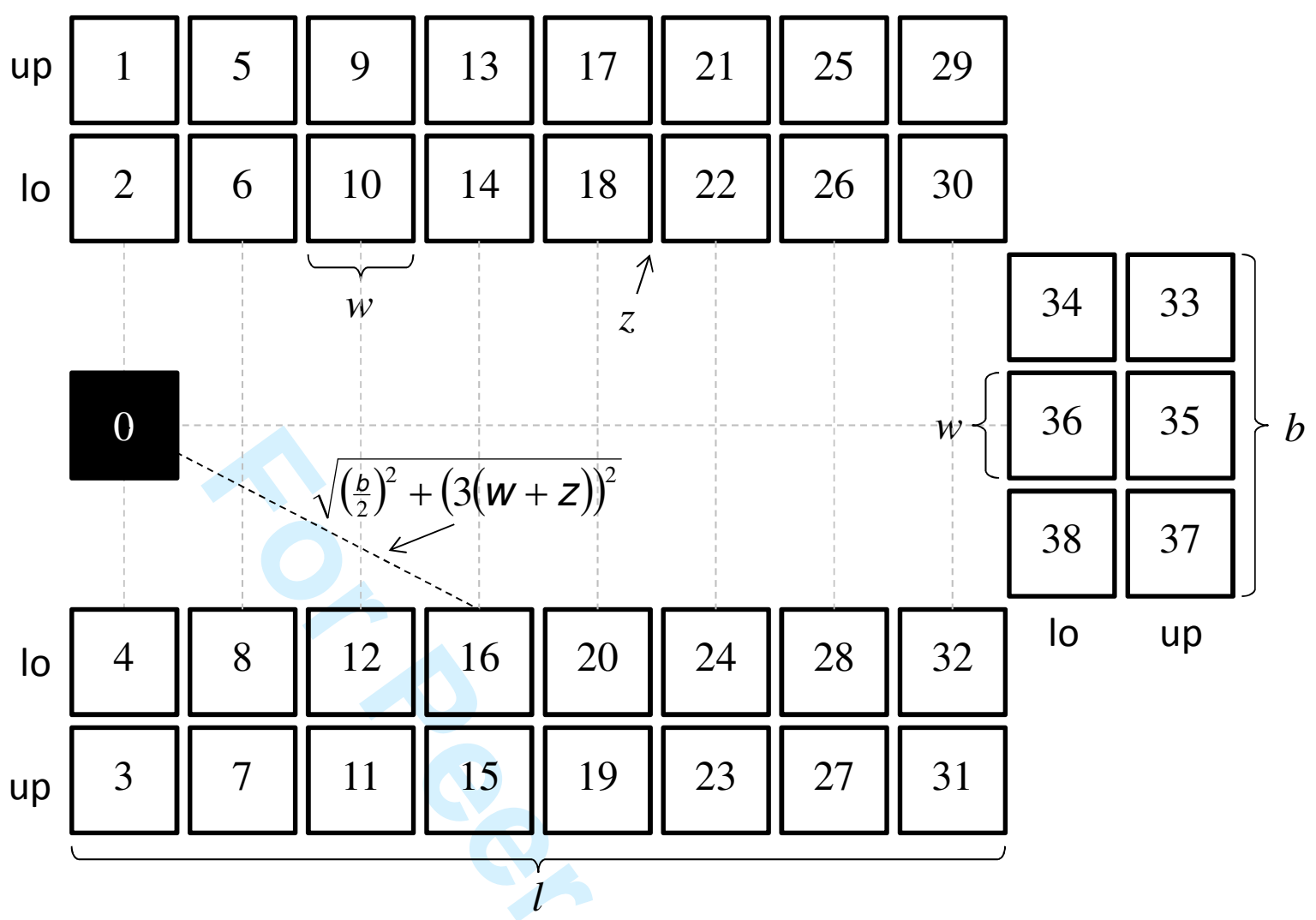

Figure 2: Example of a zone of the warehouse with 38 stillages 


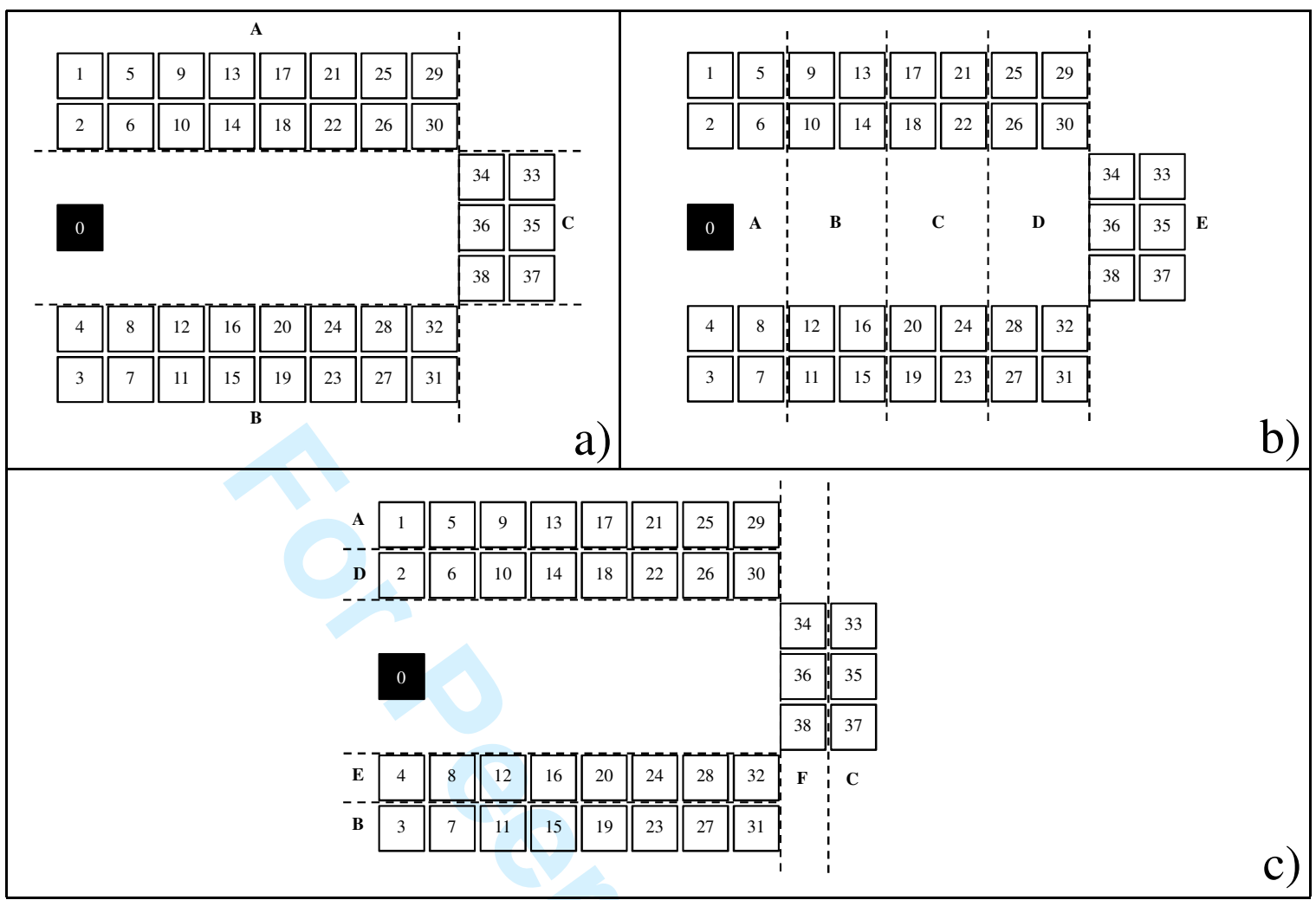

Figure 3: Illustration of the storage policies 


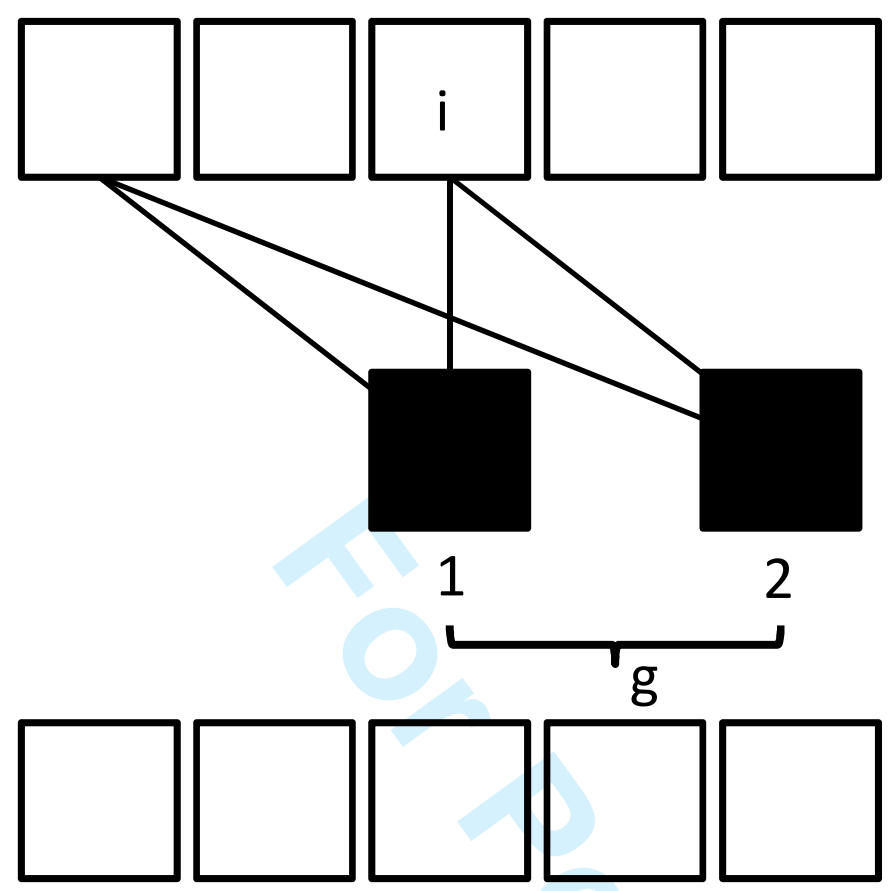

Figure A-1: A sample aisle with two alternative locations for the base 